ISSN 0103-5150

Fisioter. Mov., Curitiba, v. 24, n. 2, p. 265-274, abr./jun. 2011

Licenciado sob uma Licença Creative Commons

\title{
Análise da influência da plataforma vibratória no desempenho do salto vertical em atletas de futebol: ensaio clínico randomizado
}

\author{
Analysis of the influence of the vibrating platform in \\ vertical jump performance in soccer players: a randomized \\ clinical trial
}

\section{Weslei Luiz de Oliveira ${ }^{[a]}$, Rafael Duarte Silva ${ }^{[b]}$, Igor Junio Oliveira Custódio ${ }^{[c]}$, Sérgio Alves Machado Gregori de Barcelos ${ }^{[\mathrm{d}]}$}

[a] Fisioterapeuta, Graduado pela Faculdade de Ciências Médicas de Minas Gerais (FCMMG), Belo Horizonte, MG - Brasil, e-mail: oliveira.wl_physiotherapy@yahoo.com

[b] Fisioterapeuta, Doutorando em Ciências da Reabilitação Universidade Federal de Minas Gerais (UFMG), professor do Departamento de Fisioterapia Faculdade de Ciências Médicas de Minas Gerais (FCMMG), Belo Horizonte, MG - Brasil, e-mail: rafaelduartes@yahoo.com.br

[c] Educador Físico, Especialista em Treinamento Esportivo Universidade Federal de Minas Gerais (UFMG), preparador Físico do Departamento de Futebol de Base do Clube Atlético Mineiro (CAM), Belo Horizonte, MG - Brasil, e-mail: ijocustodio@yahoo.com.br

[d] Fisioterapeuta, Graduado pela Faculdade de Ciências Médicas de Minas Gerais (FCMMG), Belo Horizonte, MG - Brasil, e-mail: samgbarcelos@yahoo.com.br

\section{Resumo}

Objetivos: 0 objetivo deste estudo é analisar a influência da Plataforma Vibratória no desempenho do salto vertical de atletas de futebol. Materiais e métodos: A amostra foi composta de 30 atletas de futebol do sexo masculino, idade $(18,57 \pm 0,81$ anos), peso $(75,49 \pm 7,463 \mathrm{~kg})$, altura $(1,79 \pm 0,057 \mathrm{~cm})$, percentual de gordura corporal $(9.6 \pm 1,327 \%)$ e IMC $(22,89 \pm 1,354 \mathrm{~kg} / \mathrm{m} 2)$ alocados aleatoriamente em Grupo 1 (VCI) e Grupo 2 (CIQ). Valores de $\mathrm{p} \leq 0,05$ foram considerados estatisticamente significativos. Resultados: A altura do CMJSBB aumentou significativamente ( $p=0,0001$ ) após ambas as intervenções (VCI e CIQ), reduzindo também de forma significativa no período de follow-up ( $\mathrm{p}=0,0003$ ). Não houve diferença significativa entre os grupos em todos os períodos $(\mathrm{p}=0,524)$. No CMJCBB, houve um aumento significativo na altura do salto ao se comparar o grupo antes e depois das intervenções por VCI e CIQ ( $p=0,001)$ e uma diminuição significativa comparando as medidas após as intervenções com o follow-up. Em relação aos tratamentos (G1 vs G2) não houve diferença estatística entre eles 
em todos os períodos $(p=0,675)$. Discussão: De acordo com os resultados, houve diferenças em relação ao fator tempo (pré, pós e follow-up). No entanto, não foram observadas mudanças em relação aos tratamentos propostos ao G1 e ao G2. O período de exposição de quatro semanas de VCI não produziu ganhos adicionais nas características morfológicas e/ou nas propriedades neuromusculares em longo prazo no G1 quando comparados com o G2. Conclusão: 0 treinamento por VCI pode ser introduzido com fins complementares à rotina de treinamento de atletas de futebol, objetivando a melhora do desempenho muscular nos membros inferiores atribuídos de forma quantitativa na capacidade de saltar desses indivíduos.

Palavras-chave: Salto. Atleta. Futebol. Avaliação de desempenho.

\section{Abstract}

Objectives: The aim of this study is to analyze the influence of vibrating platform in the vertical jump performance in soccer players. Materials and methods: The sample consisted of 30 soccer players male, age (18:57 \pm 0.81 years), weight $(75.49 \pm 7.463 \mathrm{Kg})$, height $(1.79 \pm 0.057 \mathrm{~cm})$, body fat percentage $(9.6 \pm 1.327 \%)$ and BMI (22.89 $\pm 1.354 \mathrm{~kg} / \mathrm{m} 2)$ were randomized into Group 1 (WBV) and Group 2 (ICQ). P values $\leq 0.05$ were considered statistically significant. Results: The height of CMJUBA increased significantly $(p=0.0001)$ after both interventions (WBV and ICQ), also significantly reducing the period of follow-up ( $p=0.0003)$. There was no significant difference between groups in all periods ( $p=0.524)$. In CMJWSA, there was a significant increase in jump height when comparing the group before and after interventions by WBV and ICQ $(p=0.001)$ and a significant decrease comparing measurements after interventions with follow-up. In relation to treatment (G1 vs. G2) there was no statistical difference between them in all periods ( $p=0.675)$. Discussion: According to the results, there were differences regarding the time factor (pre, post and follow-up). However, no differences were observed in relation to proposed treatments to G1 and G2. The exposure period of four weeks of WBV produced additional gains on morphological and / or neuromuscular properties in the long term in G1 compared with G2. Conclusion: The training for WBV can be introduced for purposes additional to the routine training of soccer players, aiming at the improvement of lower limb muscle performance attributed to a quantitative leap in the ability of these individuals.

Keywords: Jump. Athlete. Soccer. Performance avaluation.

\section{Introdução}

A definição clássica de vibração foi descrita por Beer e Johnston (1) como sendo oscilações mecânicas em torno de uma posição de referência. As vibrações mecânicas que englobam diversos segmentos corporais chamadas "whole-body vibration", ou vibrações de corpo inteiro (VCI), são utilizadas atualmente na reabilitação, melhora do desempenho físico e estimulação do desenvolvimento ósseo (2).

As frequências ( $\mathrm{Hz}$ = número de eventos/ seg.) referem-se às taxas de repetição dos ciclos oscilatórios, e as amplitudes medidas (em milímetros - mm) são a extensão do deslocamento da base vibratória (3). Luo et al. (4) demonstraram que os resultados obtidos na melhora do desempenho físíco e no salto vertical são dependentes da combinação dessas propriedades, determinando a magnitude da aceleração vibratória. Diversos trabalhos vêm mostrando que a VCI, em baixas frequências e amplitudes diminutas, é um método seguro e eficaz para a melhora do desempenho físico (5-7). A maioria das plataformas vibratórias proporciona frequências de 15 a $60 \mathrm{~Hz}$ e deslocamentos de menos de 1 até $11 \mathrm{~mm}$ (8), com acelerações de até $15 \mathrm{~g}$ (aceleração gravitacional, $1 \mathrm{~g}=9,8 \mathrm{~m} / \mathrm{s}^{2}$ ). Essas variações correspondem a diferentes resultantes, porém os mecanismos de resposta dos tecidos corporais ante essas configurações são pouco compreendidos (5).

A estrutura teórica acerca da melhora no desempenho físico após a exposição vibratória pode ser atribuída a fatores neurais, tais como a sincronização das unidades motoras por meio dos moto neurônios $(6,9-11)$, potencialização do reflexo de estiramento (12-15), sinergismo da atividade muscular e alteração do sistema de inervação recíproca 
inibindo a musculatura antagonista $(6,10)$. Poucas pesquisas são voltadas às características ideais de exposição à VCI com subsequentes adaptações em longo prazo (8), e não há consenso na literatura com relação aos protocolos de exposição, que variam de 30 segundos (14) a 16 minutos (16). Em revisão sistemática, Rehn et al. (17) investigou nove estudos que avaliaram os efeitos da VCI em longo prazo. Os resultados evidenciaram um aumento estatisticamente significativo $(p<0,01)$ nas medidas de força e potência muscular nos membros inferiores. A maioria destes estudos foram ensaios clínicos aleatorizados de alta ou moderada qualidade.

No futebol, o salto vertical chega a ser o segundo gesto mais executado durante a partida e é reconhecido como uma ação motora indispensável em várias modalidades esportivas (18). A elevação do centro de massa durante o salto vertical é utilizada como forma de análise do comportamento e potência muscular, diante das características biomecânicas e fisiológicas envolvidas no movimento $(19,20)$; assim, o TSV do tipo contramovimento é utilizado por profissionais do esporte para monitorar os efeitos do treinamento e/ ou reabilitação (18). Da Silva et al. (21) mostraram um aumento da força muscular e dos parâmetros mecânicos associados à melhora do desempenho físico, correlacionando potência muscular e capacidade de saltar e correr em curtas distâncias de atletas de futebol.

Esse trabalho se faz relevante diante da heterogeneidade das amostras estudadas, da escassez de estudos controlados de longo prazo envolvendo VCI direcionados a atletas de futebol, bem como das diferenças metodológicas na mensuração dos saltos verticais e nos protocolos de exposição à VCI, o que, em suma, acarretam resultados significativamente discrepantes. Portanto, o objetivo desse estudo foi analisar a influência da Plataforma Vibratória sobre o desempenho do salto vertical em atletas de futebol.

\section{Materiais e métodos}

\section{Amostra}

Esse ensaio clínico randomizado foi desenvolvido nas dependências do Departamento de Futebol de Base do Clube Atlético Mineiro (CAM). A amostra foi composta por 30 atletas de futebol, assintomáticos, do sexo masculino, com idade $(18,57 \pm 0,81$ anos $)$, peso $(75,49 \pm 7,46 \mathrm{~kg})$, altura $(1,79 \pm 0,5 \mathrm{~cm})$, percentual de gordura corporal $(9,6 \pm 1,32 \%)$ e índice de massa corporal, IMC $\left(22,89 \pm 1,35 \mathrm{~kg} / \mathrm{m}^{2}\right)$, provenientes da categoria Júnior (Sub-20) do CAM. Foram prestados aos atletas e à comissão técnica esclarecimentos acerca dos objetivos do estudo, protocolos adotados, procedimentos inerentes, possíveis benefícios e desconfortos relativos aos experimentos. Todos os atletas voluntários assinaram um termo de consentimento, em concordância com a sua participação no estudo, e foram submetidos aos seguintes critérios de seleção:

\section{Critérios de inclusão}

1) Ser integrado à categoria Júnior do CAM.

2) Gozar de plenas condições físicas durante os testes e realização dos procedimentos.

3) Apresentar IMC entre 18,5 e $24,9 \mathrm{~kg} / \mathrm{m}^{2}$

\section{Critérios de exclusão}

1) Apresentar queixas de dores lombares ou em membros inferiores nas datas das coletas dos dados.

2) Ter sido submetido a alongamentos em MMII nas últimas 2 horas.

3) Possuir histórico de lesões musculoesqueléticas moderadas ou graves em membros inferiores e quadril nos últimos seis meses.

Os atletas incluídos no estudo foram alocados aleatoriamente em Grupo $1(\mathrm{n}=15)$ e Grupo $2(\mathrm{n}=15)$, não havendo inferência graças ao baixo intervalo de faixa etária, nem mesmo pareamento conforme IMC ou outras características antropométricas, evitando, assim, amostragem de conveniência ou viés, objetivando maior fidedignidade dos resultados e melhor representação populacional. Este estudo foi submetido à revisão e obteve parecer favorável do Comitê de Ética em Pesquisa do Hospital Universitário São José (CEP/HUSJ), processo n. 1557.0.000.418-10.

\section{Instrumentação}

\section{Bicicleta ergométrica}

As sessões de aquecimento anteriores aos testes de salto vertical (TSV) foram realizadas na sala 
de musculação do Departamento de Futebol das Categorias de Base do Clube Atlético Mineiro, em bicicletas ergométricas horizontais (Total Health ${ }^{\circledR}$ EHX 1500).

Tapete de contato

Os TSV foram realizados em um tapete de contacto Ergo Jump ${ }^{\circledR}$, a $0,1 \mathrm{~cm}$ do solo, que mede com precisão de milisegundos o período de tempo em que o indivíduo permanece sem contato durante a execução do salto $(22,19)$. 0 cálculo de conversão da elevação do centro de gravidade de milisegundos para centímetros e milímetros é obtido por meio da fórmula $\mathrm{h}=\mathrm{t} 2 \mathrm{x} \mathrm{g} \times 8 \mathrm{-1}$ (23), sendo este um sistema de medição documentado na literatura e confiável em relação à plataforma de força $(22,24)$.

Plataforma vibratória

Para exposição dos atletas do Grupo 1 à VCI, foi utilizada uma plataforma vibratória (Power Plate ${ }^{\circledR}$ Personal My3 Basic), que produziu vibrações triplanares, prioritariamente verticais a uma frequência de $35 \mathrm{~Hz}$. A amplitude vibratória foi de $\pm 4 \mathrm{~mm}$. Segundo García-Artero et al. (12), amplitudes entre 4 e $6 \mathrm{~mm}$ garantiram ativações musculares satisfatórias. A aceleração da plataforma não foi registrada por meio de acelerômetros, porém achados de Bazzet-Jones et al. (5) mostraram que amplitudes entre 4 e $6 \mathrm{~mm}$ geram acelerações em torno de $4,87 \mathrm{~g}$.

\section{Procedimentos}

\section{Procedimentos iniciais e aquecimento}

Os procedimentos iniciaram-se com a apresentação dos protocolos adotados no estudo e com a coleta dos dados relativos às características antropométricas (Idade, Peso, Altura, IMC, Percentual de Gordura Corporal) dos atletas selecionados.

A única forma de aquecimento que precedeu os TSV foi realizada em bicicletas ergométricas horizontais, durante 5 minutos em um ritmo constante de 60 rotações por minuto (RPM), com nível de resistência fixado em dois Kilopond/metro (2 KPM).
Registro do salto vertical

Os atletas foram submetidos a três baterias de TSV durante o estudo, sempre realizadas como primeira atividade do dia. Cada bateria consistiu de seis saltos verticais com intervalos periódicos de quatro semanas entre elas, realizados na primeira semana (pré-intervenção), quarta (pós-intervenção) e oitava semana (acompanhamento - follow-up). A modalidade de salto vertical empregada nos TSV foi do tipo contramovimento (countermovement jump - CMJ), três dos quais sem o balanço dos braços (CMJSBB), com as mãos posicionadas sobre as cristas ilíacas $(10,24)$, o que reduz os desvios horizontais e laterais durante o salto (2), e três com o balanço dos braços (CMJCBB), reconhecendo a influência fundamental dos membros superiores (MMSS) no desempenho final do salto vertical (22). Foi solicitado aos atletas que, a cada 20 segundos cronometrados, saltassem na maior altura possível e que, no momento da "aterrissagem", o fizessem com os pés em flexão plantar como forma de validação e padronização dos TSV, reduzindo assim as diferenças nas técnicas de salto entre os atletas. A máxima amplitude articular dos joelhos durante os TSV não foi controlada como em outros trabalhos $(2,19)$, que limitaram a amplitude em $90^{\circ}$ de flexão de joelhos. 0 desempenho do salto vertical foi registrado por meio de uma unidade portátil de computador conectada a um tapete de contato $(22,24)$, em que a média das três leituras em cada modalidade de salto (CMJSBB e CMJCBB) foi considerada como resultado dos testes.

\section{Sessões com a plataforma vibratória}

Os atletas do G1 foram posicionados no centro de oscilação da plataforma vibratória com extremidades distais dos membros inferiores equidistantes $(2$, $19,24,25)$. Diante da ausência de estudos que ofereçam um protocolo ideal de utilização da plataforma vibratória, este estudo foi baseado em protocolos semelhantes ao de outros autores que observaram, a partir de seus experimentos, importantes mudanças no desempenho muscular em populações de indivíduos treinados $(2,16,20)$. 0 protocolo de exposição à VCI foi baseado no proposto por Fagnani et al. (2), em que os atletas do G1 foram expostos à plataforma vibratória a uma frequência de $35 \mathrm{~Hz}$ e amplitude de onda de $\pm 4 \mathrm{~mm}$, devendo permanecer em posição estática e cadeia cinética fechada (CCF), com amplitude 
articular dos joelhos mantida a $90^{\circ}$ bilateralmente. Os atletas realizaram cinco séries de 60 segundos de exposição à VCI, com tempo de recuperação entre cada série igual ao de exposição vibratória (1:1). De acordo com Grigoletto et al. (26), a recuperação de um minuto se mostrou mais eficaz na força muscular cumulativa e, consequentemente, na capacidade do salto vertical. As sessões foram realizadas três vezes por semana (15 minutos de VCI semanais), durante quatro semanas ininterruptas, totalizando 12 exposições e 60 minutos de exposição à VCI.

Sessões de contração isométrica de quadríceps

Os atletas alocados no G2 realizaram com as mãos posicionadas nos quadris, cinco séries de 60 segundos de contração isométrica de quadríceps (CIQ) em CCF com tempo de recuperação igual ao de contração (1:1). Esses procedimentos foram realizados no solo, sem que os atletas tivessem nenhum contato com a plataforma vibratória durante todo o estudo. A amplitude articular dos joelhos foi controlada por meio de goniometria, permanecendo a $90^{\circ}$ de flexão durante toda a intervenção. Assim como no G1, as sessões dos atletas do G2 foram realizadas três vezes por semana, durante quatro semanas, o que totalizou períodos iguais de CIQ e VCI aos respectivos grupos.

\section{Análise estatística}

Os resultados serão apresentados como média \pm desvio-padrão e porcentagens. A normalidade na distribuição das amostras foi constatada a partir do teste de normalidade de Kolmogorov-Smirnov. Foi aplicado o two-way ANOVA com post hoc de Tukey para analisar as diferenças na altura do salto entre os grupos a partir das duas fontes de variação: tempo (pré, pós e follow-up) e intervenção (VCI e CIQ). Os testes foram aplicados separadamente no salto com e sem movimento dos braços. Valores de $\mathrm{p} \leq$ 0,05 foram considerados estatisticamente significativos. As análises foram realizadas com o auxílio do programa GraphPad Prism ${ }^{\circledR} 4.0$.

\section{Resultados}

Vinte e um atletas concluíram o estudo (G1 n = 10 e G2 $\mathrm{n}=11$ ). Em ambos os grupos, os participantes familiarizaram-se com o protocolo de exercícios durante o estudo. Grande parte dos participantes do G1 relatou prurido em MMII durante as sessões de VCI e definiu a carga vibratória como agradável e cansativa, porém não a consideraram como uma carga de trabalho extenuante. Não houve relatos de efeitos secundários adversos aos procedimentos

Não foram detectadas diferenças significativas nas características da amostra do início ao fim do estudo. As características antropométricas dos participantes incluídos no estudo encontram-se na Tabela 1.

De acordo com os testes, a altura do CMJSBB aumentou significativamente $(\mathrm{p}=0,0001)$ após ambas as intervenções (VCI e CIQ), reduzindo também de forma significativa no período de follow-up $(\mathrm{p}=$ $0,0003)$. Não se observou diferença estatisticamente significativa ( $p=0,671)$ na comparação dos saltos no período pré-intervenções com o follow-up. Em relação aos grupos (G1 vs G2), não observaram-se

Tabela 1 - Características antropométricas da amostra nos respectivos grupos

\begin{tabular}{lccccc}
\hline & \multicolumn{2}{c}{ Grupo I (VCl) } & & \multicolumn{2}{c}{ Grupo 2 (CIQ) } \\
\cline { 2 - 3 } \cline { 5 - 6 } & Média & SD & & Média & SD \\
\hline Idade & 18,5 & 0,84 & & 18,64 & 0,80 \\
Peso & 73,45 & 9,34 & & 77,34 & 4,99 \\
Altura & 1,79 & 0,07 & & 1,80 & 0,03 \\
\%G.C & 9,25 & 1,47 & & 9,93 & 1,14 \\
IMC & 22,76 & 1,53 & & 23,59 & 1,08 \\
\hline
\end{tabular}

Legenda: $\mathrm{VCI}=$ Vibração de Corpo Inteiro; CIQ = Contração Isométrica de Quadríceps; \% G.C. = Percentual de Gordura Corporal; IMC = Indice de Massa Corporal; SD = Desvio-Padrão. 
diferenças estatísticas entre eles em todos os períodos ( $p=0,524)$. 0 comportamento dos grupos durante as baterias de TSV são apresentados no Gráfico 1.

No CMJCMB, pode-se observar um resultado semelhante, havendo um aumento significativo na altura do salto ao se comparar o grupo antes e depois das intervenções por VCI e CIQ ( $p=0,001)$ e uma diminuição também significativa ao se comparar as medidas após as intervenções com o follow-up ( $\mathrm{p} \leq$ $0,05)$. Não houve diferença ao se comparar as mensurações antes da intervenção com o follow-up $(\mathrm{p}=$ 0,93). Em relação aos tratamentos (G1 vs G2), não se observou diferença estatística entre eles em todos os períodos $(\mathrm{p}=0,675)$. A média \pm desvio-padrão da altura dos saltos nos dois grupos e em ambas as modalidades podem ser observadas na Tabela 2 .

\section{Discussão}

Os resultados obtidos no presente estudo corroboram com o descrito em outros estudos que

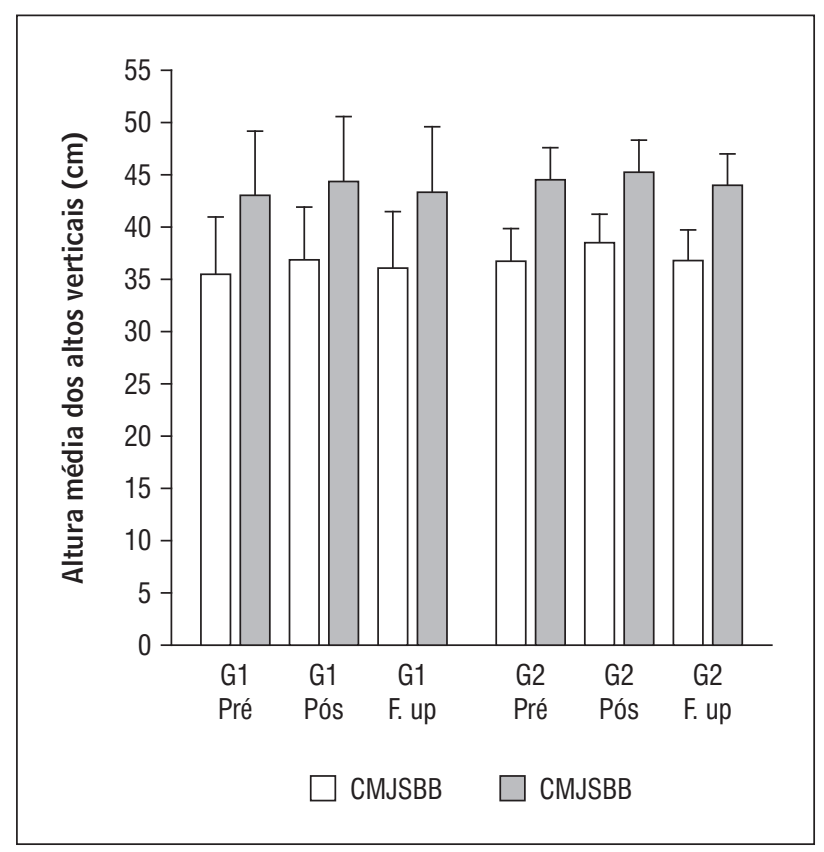

Gráfico 1 - Comportamento dos grupos durante as três baterias de testes de salto vertical em cada variável dependente

Legenda: CMJSBB = Salto contramovimento sem balanço dos braços; CMJCBB = Salto contramovimento com balanço dos braços; Pré $=$ Período anterior ao protocolo de intervenção; Pós $=$ Período posterior ao protocolo de intervenção; F. up = Follow-up, período de acompanhamento dos resultados. * Aumento estatisticamente significativo na altura dos saltos verticais. investigaram os efeitos crônicos da VCI e demonstraram melhora no desempenho do salto vertical (2, $12,16,19,20)$. Grande parte dos estudos envolvendo VCI tem resultados conflitantes. Alguns trabalhos mostram um aumento significativo no desempenho e na potência dos músculos dos MMII $(2,12,16,19$, 20, 27-32); em outros, essas diferenças não foram observadas $(20,24,33,34)$.

Observou-se melhora significativa de 4,15\% $(\mathrm{p}=0,0001)$ na altura média dos CMJSBB e de $3,52 \%$ $(\mathrm{p}=0,001)$ nos CMJCBB para o grupo de exposição à VCI (G1). Esses resultados são semelhantes aos encontrados por Paradisis e Zacharogiannis (16), que observaram aumento de 3,3\% na altura do CMJSBB em jovens expostos à $\mathrm{VCI}(30 \mathrm{~Hz}$ e $2,5 \mathrm{~mm})$ três vezes por semana durante seis semanas, em comparação ao grupo controle, que não sofreu qualquer intervenção e não obteve melhora. Também Fagnani et al. (2), ao fim de oito semanas, demonstrou melhora significativa de $8,7 \%$ na altura do CMJSBB, $13 \%$ na flexibilidade e $11,2 \%$ no pico de força dinâmica $(p<0,05)$, de 24 atletas submetidas à VCI ( $35 \mathrm{~Hz}, \mathrm{e} \pm 4 \mathrm{~mm})$, em posição articular de $90^{\circ}$ de flexão de joelhos.

De acordo com os resultados, observou-se diferença significativa em relação ao fator tempo (pré, pós e follow-up). No entanto, não houve diferença em relação aos tratamentos propostos ao Grupo 1 (VCI) e ao Grupo 2 (CIQ). Essa relação de ausência de melhora adicional decorrente de diferentes tratamentos também foi reportada previamente na literatura. Em 2003, De Ruiter et al. analisaram os efeitos de 11 semanas de treinamento por VCI (30 $\mathrm{Hz}$ e $8 \mathrm{~mm}$ ) sobre o desempenho do salto vertical e força isométrica de 20 indivíduos. Comparando com o grupo que realizou os mesmos exercícios fora da plataforma vibratória, não se observaram diferenças significativas em nenhum dos parâmetros referidos. Na revisão de Nordlund e Thorstensson (35), foram avaliados 12 estudos de uso crônico de VCI (mínimo de uma semana), em que cinco desses $(n=146)$ tinham delineamento metodológico de ensaios clínicos com grupo controle, realizando exercícios sem a exposição vibratória, e concluíram que quatro desses cinco estudos não apresentaram diferenças significativas entre os grupos, com resultados de melhora no desempenho do salto vertical variando entre $4,5 \% \mathrm{e}$ $16 \%$. Luo et al. (4), em sua revisão, propôs que os resultados inconsistentes são decorrentes de trabalhos que apresentam diferenças nos protocolos e falhas metodológicas importantes, além de apresentarem 
Tabela 2 - Média \pm desvio-padrão da altura dos saltos com e sem o balanço dos braços nos grupos G1 e G2

\begin{tabular}{lccccc}
\hline & \multicolumn{2}{c}{ VCI (G1) } & & \multicolumn{2}{c}{ CIQ (G2) } \\
\cline { 2 - 3 } \cline { 5 - 6 } & CMJSBB & CMJCBB & & CMJSBB & CMJCBB \\
\hline Pré & $35,49 \pm 5,46$ & $42,94 \pm 6,33$ & & $36,87 \pm 2,77$ & $44,45 \pm 2,86$ \\
Pós & $36.96 \pm 4,89$ & $44,45 \pm 6,11$ & & $38,42 \pm 2,71$ & $45,16 \pm 3,19$ \\
Follow-up & $36,15 \pm 5,12$ & $43,42 \pm 6,37$ & & $36,75 \pm 2,87$ & $43,86 \pm 3,06$ \\
\hline
\end{tabular}

Legenda: VCI (G1) = Grupo 1 - Vibração de Corpo Inteiro; CIQ (G2) = Grupo 2 - Contração Isométrica de Quadríceps; Pré = Período anterior ao protocolo de intervenção; Pós = Período posterior ao protocolo de intervenção; Follow-up = Período de acompanhamento posterior ao protocolo de intervenção; CMJSBB = Salto contramovimento sem balanço dos braços; CMJCBB = Salto contramovimento com balanço dos braços.

amostras e propriedades vibratórias discrepantes quando se trata dos indivíduos expostos a VCI em comparação ao grupo controle.

Foi constatado um decréscimo aproximado na altura dos saltos verticais entre os grupos no período de follow-up, indicando que ambos os grupos perderam em desempenho muscular, quatro semanas após o período de intervenção. Bazzet-Jones et al. (5) relatou que os efeitos positivos da VCI no desempenho físico e muscular são de natureza transitória, durando entre cinco e dez minutos após a VCI, limitando-se, assim, a adaptações de curto prazo, não perdurando por longos períodos.

Ao que parece, o período de exposição de quatro semanas de VCI não produziu ganhos adicionais nas características morfológicas e/ou nas propriedades neuromusculares em longo prazo no G1, quando comparado com o G2. A ausência de melhora adicional na altura do salto no grupo de exposição em relação ao controle nos remete às bases fisiológicas da adaptação ao treinamento, onde a primeira fase de adaptação é caracterizada por uma facilitação dos fatores neurais aos quais se atenuam como efeitos agudos da VCI, enquanto que alterações na estrutura morfológica do músculo necessárias para melhora do rendimento físico podem levar de meses a anos (16).

Gomes et al. (36) reportaram que o melhor desempenho do salto vertical parece estar envolvido com a maior duração da fase concêntrica do movimento. Este mesmo autor analisou as características cinéticas e cinemáticas de 18 atletas de duas modalidades esportivas distintas durante o CMJCBB e CMJSBB, e não encontraram diferenças significativas na altura máxima do salto vertical entre eles.
Outros trabalhos mostraram que a velocidade de saída do solo é um fator determinante para a altura do salto e que esta velocidade depende, sobretudo, da potência muscular (37). Vale ressaltar que, além do disposto, o CMJ tem características peculiares, como o maior deslocamento angular. Tal característica evocaria de forma menos eficiente o reflexo de estiramento, comprometendo os efeitos de vibração pelo esforço voluntário $(19,22)$. Outra hipótese reportada em estudos sugere que frequências vibratórias entre 20 e $35 \mathrm{~Hz}$ não têm influência no desempenho do salto vertical e no desenvolvimento da força "explosiva" dos MMII $(5,36)$. Ronnestad (38) não encontrou diferenças signifivas na produção média de potência máxima muscular durante a realização do CMJ, quando utizadas frequência de vibração de 20 e $35 \mathrm{~Hz}$, em comparação com nenhuma vibração no grupo controle. Bazzet-Jones et al. (5) relatou, em seus experimentos, que frequências vibratórias entre $30 \mathrm{~Hz}(1,81 \mathrm{~g}, 2-4 \mathrm{~mm})$ e $35 \mathrm{~Hz}$ (4,87 g, 4-6 mm) não provocaram mudanças no desempenho dos saltos verticais, independentemente de aceleração ou amplitude. Mileva et al. (39) mostrou que baixas frequências de VCI $(30 \mathrm{~Hz})$ induziram a plasticidade neural no SNC, aumentando a excitabilidade da via corticoespinhal, suportando a probabilidade de adaptações neurais; porém, o estudo de Colson et al. (40) contestou tais achados e mostrou que a ativação muscular voluntária dos extensores do joelho permaneceu inalterada pela VCI e que, independentemente da intervenção, a contração isométrica voluntária máxima foi reduzida significativamente.

A estimulação sensorial por meio de vias aferentes é a base da atividade muscular em treinamentos 
de VCI em curto prazo, atribuindo a ela meios de facilitação neural e adaptação neuromuscular ligada à melhora do $\operatorname{CMJ}(6,16,20,24,41)$. Essa relação ainda é pouco compreendida, e sem relatos consistentes de como esse mecanismo provocaria efeitos positivos sustentados em longo prazo sobre tudo no salto vertical (35), havendo inclusive evidências que indicam a VCI como um precursor inibitório do recrutamento de unidades motoras em situações de exposição prolongada $(31,33,41)$.

Neste estudo não foi analisado se a posição e a função tática do atleta em campo influenciariam na altura dos saltos verticais. Esse fator não é descrito na literatura. Não foram controladas a periodização e a intensidade dos treinamentos aos quais os atletas eram submetidos ao longo do estudo, o que pode ter impacto sobre os resultados. Uma avaliação eletromiográfica do padrão de ativação muscular poderia fornecer parâmetros mais consistentes em relação às adaptações neuromusculares ao longo dos procedimentos. Futuros estudos devem levar em consideração essas variáveis e mensurar qual é sua real influência da VCI no desempenho do salto vertical.

\section{Conclusão}

Concluímos que o treinamento por VCI de longo prazo contribui para a melhora da capacidade muscular em membros inferiores de atletas de futebol, podendo ser introduzido com fins complementares à rotina de treinamento desses atletas, visando a um maior desempenho do salto vertical, gesto altamente relacionado com a prática esportiva em questão.

\section{Referências}

1. Beer ER, Johnston ER. Mechanical vibrations. In: Mechanics for Engineers. Toronto: McGraw-Hill Book Company; 1987. p. 838-88.

2. Fagnani F, Giombini A, Di Cesare A, Pigozzi F, Di Salvo V. The effects of a whole-body vibration programon on muscle performance and flexibility in female athletes. Am J Phys Med Rehabil. 2006;85(12):956-62.

3. Rauch, F. Vibration therapy. Dev Med Child Neurol. 2009;51(Suppl.4):166-8.
4. Luo J, McNamara B, Moran K. The use of vibration training to enhance muscle strength and power. Sports Medic. 2005;35(1):23-41.

5. Bazett-Jones DM, Finch HW, Dugan EL. Comparing the effects of various whole-body vibration accelerations on counter-movement jump performance. J Sports Sci Med. 2008;7:144-50.

6. Cardinale $\mathrm{M}$, Bosco $\mathrm{C}$. The use of vibration as an exercise intervention. Exerc Sport Sci Rev. 2003;31(1): 3-7.

7. Cardinale M, Wakeling J. Whole body vibration exercise: are vibrations good for you? Br J Sports Med. 2005;39(9):585-9.

8. Dolny D, Reyes F. Whole body vibration exercise: training and benefits. Curr Sports Med Rep. 2008; $7(3): 152-7$.

9. Rittweger J, Ehrig J, Just K, Mutschelknauss M, Kirsch KA, Felsenberg D. Oxygen Uptake in whole-body vibration exercise: influence of vibration frequency, amplitude, and external load. Int J Sports Med. 2002; 23(6):428-32.

10. Bullock N, Martin DT, Ross A, Rosemond CD, Jordan MJ, Marino FE. Acute effect of whole-body vibration sprint and jumping performance in elite skeleton athletes. J Strength Cond Res. 2008;22(4):1371-4.

11. Abercromby AF, Amonette WE, Paloski WH, Hinman MR. Effect of knee flexion angle on neuromuscular responses to whole-body vibration. In: Abstract presented at NSCA National Conference, July 2005. [acesso em 20 mar. 2011]. Disponível em: http:// journals.lww.com/nsca-jscr/Citation/2005/11000/ NSCA_2005_Conference_Abstracts.40.aspx.

12. García-Artero E, Ortega Porcel FB, Ruiz JR, Carreño Gálvez F. Entrenamiento vibratorio. Base Fisiológica y Efectos Funcionales. 2006;15(2):78-86.

13. Nishihira Y, Iwasaki T, Hatta A, Wasaka T, Kaneda T, Kuroiwa K, et al. Effect of whole body vibration stimulus and voluntary contraction on motoneuron pool. Adv Exerc Sport Physiol. 2002;8(4):83-6.

14. Cormie P, Deane RS, Triplett NT, Mcbride JM. Acute effects of effects of whole-body vibration on muscle activity, strength, and power. J Strength Cond Res. 2006;20(2):257-61. 
15. Da Silva ME, Vaamonde Martín DM, Padullés JM. Efectos del entrenamiento con vibraciones mecánicas sobre la performance neuromuscular. Apunts. Educación Física y Deportes. 2006;(84):39-47.

16. Paradisis G, Zacharogiannis E. Effects of whole-body vibration training on sprint running kinematics and explosive strength performance. J Sports Science Med. 2007;6:44-49.

17. Rehn B, Lidström J, Skoglund J, Lindström B. Effects on leg muscular performance from whole-body vibration exercise: a systematic review. Scand J Med Sci Sports. 2007;17(1):2-11.

18. Silva P, Oliveira G. Análise biomecânica e neuromuscular da musculatura extensora do trem inferior no salto de impulsão vertical. [Revista online] efdeportes.com - Buenos Aires. 2003 [acesso em: 15 des. 2010];9(67). Disponível em: http://www.efdeportes.com.

19. Di Giminiani RD, Tihanyi J, Safar S, Scrimaglio R. The effects of vibration on explosive and reactive strength when applying individualized vibration frequencies. J Sports Sciences. 2009;27(2):169-77.

20. Delecluse C, Roelants M, Verschueren S. Strength increases after whole-body vibration compared with resistance training. Med Sci Sports Exerc. 2003;35(6): 1033-41.

21. Da Silva ME, Padullés JM, Núñez VM, Vaamonde DM, Viana B, Renedo M, et al. Efectos agudos del entrenamiento con vibraciones mecánicas sobre el tiempo de carrera en corta distancia en jugadores de fútbol. Med Ejercicio. 2006;21(1-2):39-47.

22. Cochrane DJ, Stannard SR. Acute whole-body vibration training increases vertical jump and flexibility performance in elite female field hockey players. $\mathrm{Br}$ J Sports Med. 2005;39(11):860-5.

23. Ugrinowitsch C, Barbanti VJ, Gonçalves A, Peres BA. Capacidade dos testes isocinéticos em predizer a "performance" no salto vertical em jogadores de voleibol. Rev Paul Educ Fís. 2000;14(2):172-83.

24. Cochrane DJ, Legg SJ, Hooker MJ. The short-term effect of whole body vibration training on vertical jump, sprint and agility performance. J Strength Cond Res. 2004;18(4):828-32.
25. Margarita G, Paradisis G, Tziortzis S. Acute effects of various whole-body vibration frequencies on squat jump in men and women: 1638. Medicine \& Science in Sports \& Exercise. 2009;41(5):79.

26. Grigoletto ME, Vaamonde DM, Castillo E, Poblador MS, García-Manso JM, Lancho JL. Acute and cumulative effects of different times of recovery from whole body vibration exposure on muscle performance. J Strength Cond Res. 2009;23(7):2073-82.

27. Bosco C, Cardinale M, Colli R, Tihanyi J, Von Duvillad SP, Viru A. The influence of whole body vibration on jumping ability. Biology of Sport. 1998;15(3):157-64

28. Roelants M, Delecluse C, Verschueren SM. Effects of 24 weeks whole body vibration training on body compositionand muscle strength in untrained females. Int J Sports Med. 2004;25(1):1-5.

29. Roelants M, Delecluse C, Verschueren SM. Wholebody-vibration training increases knee-extension strength and speed of movement in older women. J Am Geriatr Soc. 2004;52(6):901-8.

30. Russo CR, Lauretani F, Bandinelli S, Bartali B, Cavazzini C, Guralnik JM, et al. High frequency vibration training increases muscle power in postmenopausal women. Arch Phys Med Rehabil. 2003; 84(12):1854-7.

31. Torvinen S, Kannu P, Sievanen H, Jarvinen TA, Pasanen M, Kontulainen S, et al. Effect of a vibration exposure on muscular performance and body balance. Randomized cross-over study. Clin Physiol Funct Imaging. 2002;22(2):145-52.

32. Torvinen S, Kannu P, Sievanen H, Jarvinen TA, Pasanen M, Kontulainen S, et al. Effect of 8-month vertical whole body vibration on bone, muscle performance, and body balance: a randomized controlled study. J Bone Miner Res. 2003;18(5):876-84.

33. De Ruiter CJ, Van Raak SM, Schilperoort JV, Hollander AP, de Haan A. The effects of 11 weeks of whole body vibration training on jump height contractile properties and activation of human knee extensor. Eur J Appl Physiol. 2003;90(5-6):595-600.

34. Schlumberger A, Salin D, Schmidtbleicher D. Strength training with superimposed vibrations. Sportverletz Sportschaden. 2001;15(1):1-7. 
35. Nordlund MM, Thorstensson A. Strength training effects of whole-body vibration. Scand J Med Sci Sports. 2007;17(1):12-7.

36. Gomes MM, Pereira G, Freitas PB, Barela JA. Características cinemáticas e cinéticas do salto vertical comparação entre jogadores de futebol e basquetebol. Rev Bras Cineantropom Desempenho Hum. 2009; 11(4):392-9.

37. Lees A, Vanrenterghem J, De Clercq D. Understanding how an arm swing enhances performance in the vertical jump. J Biomech. 2004;37(12):1929-40.

38. Ronnestad BR. Acute efeccts of varius wbv frequencies on lower-body power in training and untrained subject. J Strength Cond Res. 2009;23(4):1309-15.
39. Mileva KN, Bowtell JL, Kossev AR. Effects of low-frequency whole-body vibration on motor-evoked potentials in healthy men. Exp Physiol. 2009;94(1):103-16.

40. Colson SS, Petit PD, Hébreard L, Tessaro J, Pensini M. Whole body vibration does not enhance muscle activation. Int J Sports Med. 2009;30(12):841-4.

41. Stewart JA, Cochrane DJ, Morton RH. Differential effects of whole body vibration durations on knee extensor strength. J Sci Med Sport. 2009;12(1):50-3.

Recebido: 28/12/2010

Received: 12/28/2010

Aprovado: 18/03/2011

Approved: 03/18/2011 